\title{
AGENTES ANTINEOPLÁSICOS BIORREDUTÍVEIS: UMA NOVA ALTERNATIVA PARA O TRATAMENTO DE TUMORES SÓLIDOS
}

\author{
Renata Barbosa de Oliveira \\ Departamento de Química, Instituto de Ciências Exatas, Universidade Federal de Minas Gerais, Av. Antônio Carlos, 6627, \\ 31270-901 Belo Horizonte - MG \\ Ricardo José Alves* \\ Química Farmacêutica, Faculdade de Farmácia, Universidade Federal de Minas Gerais, Av. Olegário Maciel, 2360, 30180-112 \\ Belo Horizonte - MG
}

Recebido em 17/8/01; aceito em 27/2/02

\begin{abstract}
BIOREDUCTIVE ANTINEOPLASTIC AGENTS: A NEW APPROACH TO THE TREATMENT OF SOLID TUMORS. A problem often encountered in cancer therapy is the presence of tumor cell subpopulation that are resistant to treatment. Solid tumors frequently contain hypoxic cells that are resistant to killing by ionizing radiation and also by many chemotherapeutic agents. However, these hypoxic cells can be exploited for therapy by non-toxic hypoxic-activated prodrugs. Bioreductive drugs require metabolic reduction to generate cytotoxic metabolites. This process is facilitated by appropriate reductases and the lower oxygen conditions present in solid tumors. The unique presence of hypoxic cells in human tumors provides an important target for selective cancer therapy.
\end{abstract}

Keywords: solid tumor; hypoxic cells; bioreductive prodrugs.

\section{INTRODUÇÃO}

O câncer é basicamente uma doença de células, caracterizada por um desvio dos mecanismos de controle que dirigem a proliferação e a diferenciação celulares. As células que sofreram transformação neoplásica proliferam excessivamente e formam tumores locais que podem comprimir ou invadir estruturas normais adjacentes ${ }^{1}$.

Progressos importantes na quimioterapia foram registrados na área da biologia molecular e celular, o que facilitou, juntamente com o maior entendimento do mecanismo de ação de muitas substâncias, a aplicação mais racional dos quimioterápicos e o planejamento de novos fármacos. Muitas das substâncias citotóxicas mais potentes atuam em fases específicas do ciclo celular e, consequientemente, só exercem a sua atividade contra células que se encontram em processos de divisão ${ }^{2}$. Sendo assim, as neoplasias malignas humanas que, atualmente, são mais suscetíveis ao tratamento quimioterápico e, freqüentemente, curadas, são aquelas que possuem alta porcentagem de células em processo de divisão. Os tumores sólidos, que apresentam divisão celular relativamente lenta, tais como os carcinomas de pulmão, cólon e mama, constituem mais de $90 \%$ de todos os tipos de câncer do homem. Estes tipos de neoplasias, em geral, respondem pouco aos agentes quimioterápicos existentes e o tratamento curativo, utilizando qualquer umas das modalidades terapêuticas (cirurgia, radioterapia, quimioterapia, imunoterapia e fototerapia dinâmica) ou mesmo a combinação das diversas modalidades, é extremamente difícil ${ }^{3}$. Além disso, uma outra limitação da quimioterapia é que a maioria dos fármacos antineoplásicos é altamente tóxica para o paciente e deve ser administrada com extremo cuidado ${ }^{2,4}$.

\section{CARACTERÍSTICAS DOS TUMORES SÓLIDOS}

Ao contrário da percepção popular, a estrutura de um tumor não consiste, simplesmente, em um aglomerado de células em constante

*e-mail: ricky@dedalus.lcc.ufmg.br proliferação. As células neoplásicas freqüentemente ocupam menos da metade do volume total do tumor. Os vasos sangüíneos que se entrelaçam dentro da massa tumoral preenchem 1 a $10 \%$ do volume do tumor. O espaço restante é preenchido por matriz rica em colágeno - o interstício - que envolve as células neoplásicas e pode separá-las da vascularizaçãa $0^{5}$.

Inicialmente, o abastecimento sangüíneo do tumor é realizado através da vascularização existente na região. Durante o crescimento do tumor, alguns dos vasos sangüíneos pré-existentes são obstruídos ou comprimidos. Posteriormente, pequenos vasos sangüíneos começam a surgir (neovascularização) e estes ramificam-se excessivamente, são tortuosos e seguem em direções imprevisíveis. Conseqüentemente, algumas áreas do tumor são bem irrigadas, outras têm pouca ou nenhuma vascularização, o que faz com que o aporte de oxigênio e nutrientes para algumas regiões do tumor seja muito menos eficiente do que nos tecidos normais. As células localizadas próximas aos vasos sangüíneos (periferia do tumor) são células bem oxigenadas. As células localizadas no centro do tumor e, portanto, distantes da vascularização, são células anóxicas e necróticas. Finalmente, as células existentes entre estas duas regiões são conhecidas como células em hipóxia, pois estão localizadas em uma região de baixa concentração de oxigênio (Figura 1$)^{5-9}$.

\section{Resistência das células tumorais em hipóxia ao tratamento do câncer}

Sabe-se que as células com oxigenação normal são mais sensíveis aos efeitos da radiação ionizante do que as células em condições de hipóxia. Isto ocorre porque as moléculas de $\mathrm{O}_{2}$ reagem rapidamente com os radicais livres produzidos pela radiação, originando radicais altamente reativos, tais como os radicais peroxila (ROO-), hidroxila (HO.) e superóxido $\left(\mathrm{O}_{2}^{-}\right)$, que provocam a destruição das biomoléculas com a consequiente morte celular ${ }^{10,11}$. Portanto, como as células tumorais em hipóxia localizam-se em regiões de baixa concentração de oxigênio, tais células tornam-se extremamente resistentes à radioterapia $^{12,13}$. 


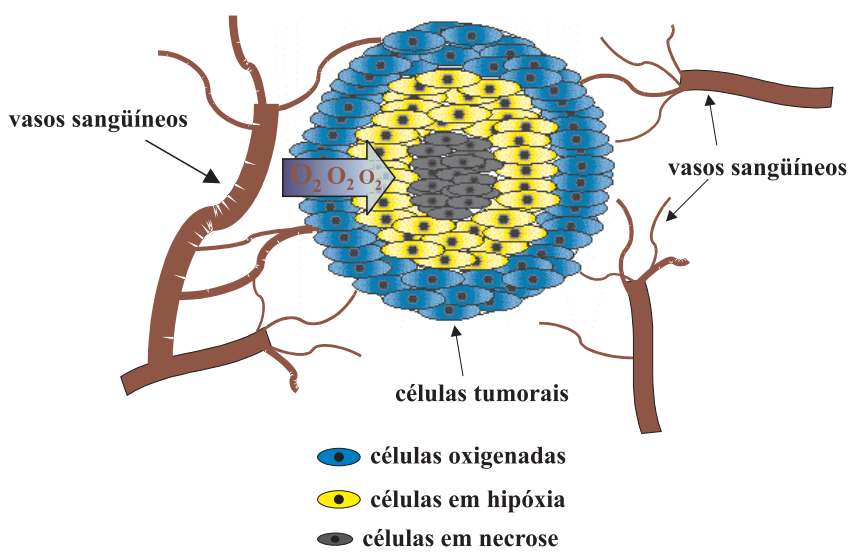

Figura 1. Secção transversal de um tumor sólido, monstrando a diminuição da concentração de oxigênio em relação à distância capilar (adaptado da referência 3)

Um dos fatores que explica a resistência dos tumores sólidos à quimioterapia é a dificuldade do fármaco em alcançar as células em hipóxia. O agente quimioterápico deve vencer várias barreiras para conseguir alcançar as células em hipóxia em concentrações terapêuticas: 1) atingir os vasos sangüíneos do tumor; 2) atravessar a parede destes vasos em direção ao interstício e 3) difundir pelo interstício em direção às células em hipóxia (Figura 2) 2,6,8 $^{5,}$

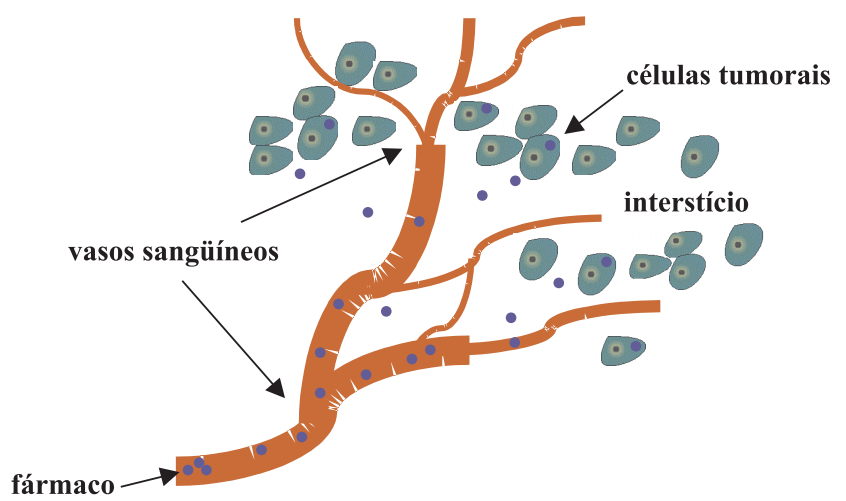

Figura 2. Barreiras que o agente antineoplásico deve vencer para alcançar as células em hipóxia (adaptado da referência 5)

Além disso, as células em hipóxia, apesar de serem capazes de se dividir, apresentam ciclo celular prolongado e permanecem indefinidamente em repouso (fase $G_{0}$ ) ${ }^{4,14}$. Portanto, pode-se esperar que estas sejam relativamente resistentes aos agentes quimioterápicos utilizados atualmente, uma vez que os mesmos são, em sua maioria, fármacos que interferem na divisão celular e atuam, principalmente, sobre células em rápida divisão ${ }^{4}$.

\section{AGENTES ANTINEOPLÁSICOS BIORREDUTÍVEIS}

Em 1972, Sartorelli e colaboradores ${ }^{15}$ levantaram a hipótese de que células em hipóxia poderiam apresentar maior capacidade de redução do que as células normalmente oxigenadas. Foi proposto, então, que esta característica das células em hipóxia poderia ser explorada no desenvolvimento de agentes antineoplásicos, os quais só se tornariam citotóxicos após ativação metabólica pelas nitrorredutases celulares ${ }^{15}$. Estes agentes antineoplásicos biorredutíveis são pró-fármacos, classificados como bioprecursores. Os bioprecursores são substâncias inativas (pró-fármacos) que, in vivo, sofrem metabolização, geralmente pelo sistema redox celular, dando origem à uma nova substância na forma ativa (fármaco $)^{16,17}$ :

\section{Pró-Fármaco Biorredução Biorredutível \\ Fármaco Reduzido}

Propriedades fundamentais apresentadas pelos agentes antineoplásicos biorredutíveis

Três propriedades são fundamentais para o sucesso da atividade dos agentes antineoplásicos seletivos para células em hipóxia: solubilidade e difusibilidade adequadas, redução a espécies reativas somente nas regiões de células em hipóxia e atividade apenas das espécies reduzidas ${ }^{18}$.

O mecanismo de ação proposto para os agentes alquilantes biorredutíveis sugere que a ativação bioquímica de tais substâncias é dependente do seu potencial de redução ${ }^{18,19}$. Estudos têm sugerido que, para a maioria dos compostos nitroaromáticos, a faixa de potencial de redução monoeletrônica $\left(\mathrm{E}_{1 / 2} \mathrm{ArNO}_{2} / \mathrm{ArNO}_{2}{ }^{\circ}\right)$ ideal seria de -400 a $-200 \mathrm{mV}$ vs ENH, em meio prótico, em $\mathrm{pH} 7\left(5 \times 10^{-3} \mathrm{M}\right.$ tampão fosfato $)^{18,20}$. Para a atividade in vivo são aceitáveis valores um pouco menores ${ }^{18}$.

\section{Principais classes de agentes biorredutíveis}

O conceito de ativação biorredutiva de substâncias em células em hipóxia tem sido extensivamente estudado ${ }^{3,5-7,9,10,12-15,18-25}$ e três classes principais são bem conhecidas. A primeira é a dos antibióticos contendo uma função quinona, dos quais a mitomicina $\mathrm{C}$ (1) é o protótipo $^{21}$.<smiles>CO[C@]12C3N[C@@H]3CN1C1=C(C(=O)C(N)=C(C)C1=O)[C@@H]2COC(N)=O</smiles>

(1)

A segunda classe é a dos nitroimidazóis, os quais foram inicialmente utilizados como radiossensibilizadores, mas que também apresentam toxicidade preferencial para células em hipóxia. Os principais representantes desta classe são o metronidazol (2) e o misonidazol (3) ${ }^{10}$.<smiles>Cc1ncc([N+](=O)[O-])n1CCO</smiles>

(2)

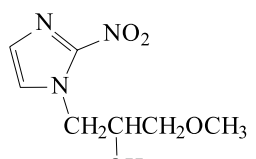

(3) $\mathrm{OH}$
A terceira é a classe dos di- $N$-óxidos de benzotriazinas, representada pela tirapazamina (1,4-di- $N$-óxido-3-amino-1,2,4benzotriazina, SR 4233) (4) $)^{25}$.<smiles>Nc1n[n+]([O-])c2ccccc2[n+]1[O-]</smiles> 


\section{Quinonas}

Quinonas apresentando porções alquilantes em sua estrutura representam uma classe de substâncias que tem recebido considerável interesse como antitumorais desde o final dos anos 50. As enzimas mais importantes envolvidas na ativação dessas quinonas são a NADPH:citocromo $\mathrm{P} 450$ redutase e a NAD(P)H:quinona oxidorredutase (DT-diaforase) ${ }^{26}$.

A mitomicina C (1) foi isolada, por Wakaki e colaboradores, de culturas de Streptomyces caespitosus em $1958^{27}$. Quatro mitomicinas (Figura 3) são de origem natural. Todas elas são antibióticos efetivos contra bactérias Gram positivas e Gram negativas, mas somente a mitomicina $\mathrm{C}$ (1) e a porfiromicina (7) apresentam atividade antitumoral $^{26}$<smiles>[X]C1=C(C)C(=O)C2=C(C1=O)C(COC(N)=O)C1([Y])C3C(CN21)N3[Z]</smiles>

\begin{tabular}{llll} 
& \multicolumn{1}{c}{$\mathrm{X}$} & \multicolumn{1}{c}{$\mathrm{Y}$} & \multicolumn{1}{c}{$\mathrm{Z}$} \\
Mitomicina A (5) & $\mathrm{OCH}_{3}$ & $\mathrm{OCH}_{3}$ & $\mathrm{H}$ \\
Mitomicina B (6) & $\mathrm{OCH}_{3}$ & $\mathrm{OH}$ & $\mathrm{CH}_{3}$ \\
Mitomicina C (1) & $\mathrm{NH}_{2}$ & $\mathrm{OCH}_{3}$ & $\mathrm{H}$ \\
Porfiromicina (7) & $\mathrm{NH}_{2}$ & $\mathrm{OCH}_{3}$ & $\mathrm{CH}_{3}$
\end{tabular}

Figura 3. Estruturas das quatro mitomicinas de origem natural ${ }^{31}$
Vários trabalhos têm demonstrado que a ativação redutiva da mitomicina $\mathrm{C}$ (1) resulta na formação de intermediários reativos que se ligam ao ADN por meio de ligações cruzadas entre duas fitas complementares (ligação interfita) ${ }^{28,29}$. Considera-se que a ativação biológica da mitomicina $\mathrm{C}$ (1) ocorre após redução monoeletrônica, com a formação de um ânion radical, ou redução bieletrônica, com a formação da hidroquinona correspondente ${ }^{24,28}$. Através da análise do mecanismo de biorredução da mitomicina $\mathrm{C}(\mathbf{1})$, representado na Figura 4, pode-se observar que o nitrogênio heterocíclico apresenta um caráter de nitrogênio de amida devido à conjugação do par de elétrons não ligante do nitrogênio com a carbonila da quinona. Entretanto, após sofrer biorredução, este nitrogênio passa a ter um caráter de amina, apresentando maior poder nucleofílico e favorecendo, então, a expulsão do grupo metoxila. A partir daí é desencadeada uma série de etapas, resultando na formação de espécies altamente reativas responsáveis pela atividade da mitomicina $C(\mathbf{1})^{17,30}$.

A mitomicina $\mathrm{C}$ (1) tem sido utilizada em quimioterapia no tratamento de vários tipos de tumores sólidos, mas seu uso é limitado em razão dos efeitos colaterais, tais como mielossupressão e danos gastrintestinais ${ }^{26}$. Em razão disto, vários análogos da mitomicina $\mathrm{C}$ (1) têm sido sintetizados buscando diminuir a toxicidade e aumentar a eficácia ${ }^{31-34}$.

A porfiromicina (7) foi a primeira substância antitumoral submetida à triagem clínica como citotoxina seletiva para células em hipóxia ${ }^{35}$. A atividade antitumoral da porfiromicina (7) vem sendo estudada desde $1967^{36}$. Atualmente, ela encontra-se em fase III de triagem clínica, em associação com a radioterapia, no tratamento de carcinoma de células escamosas de cérebro e pescoço ${ }^{37}$. Apesar da maior eficácia da porfiromicina (7) em relação à mitomicina $\mathrm{C}$ (1), seu uso clínico também é limitado pela mielossupressão que provo$\mathrm{ca}^{26}$.

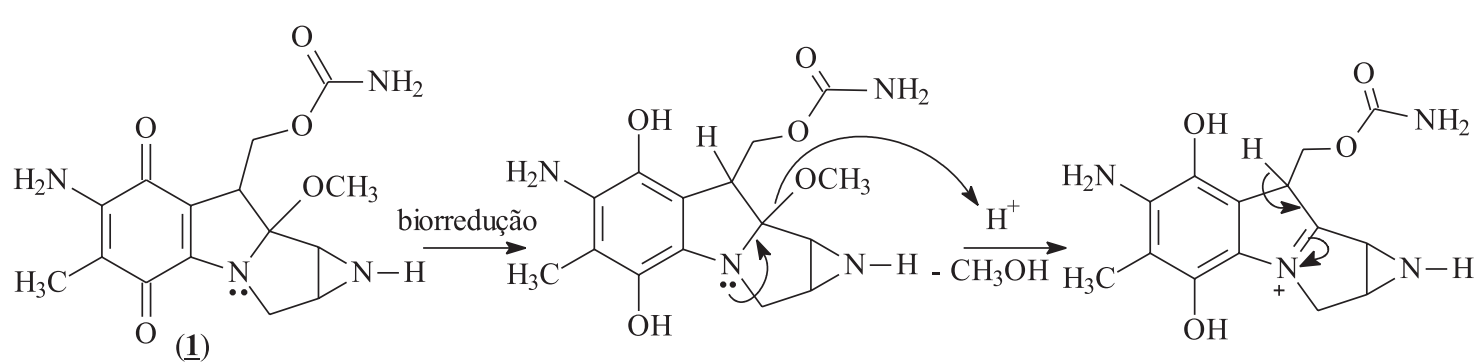

(1)

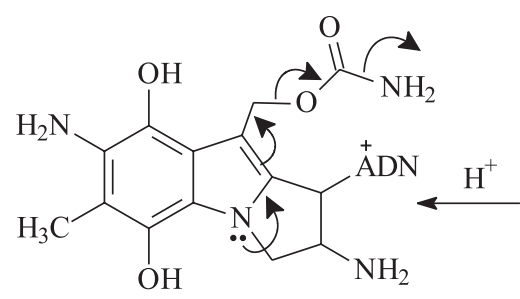

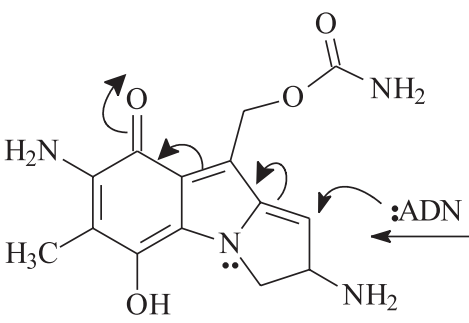

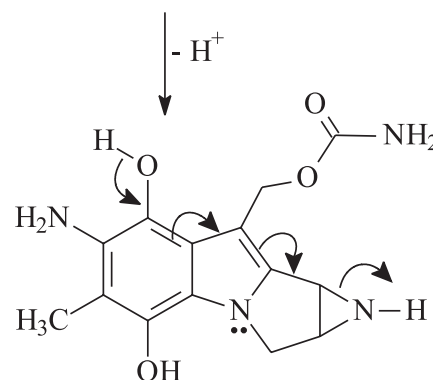<smiles>[Y]C(=O)O[Na]</smiles>

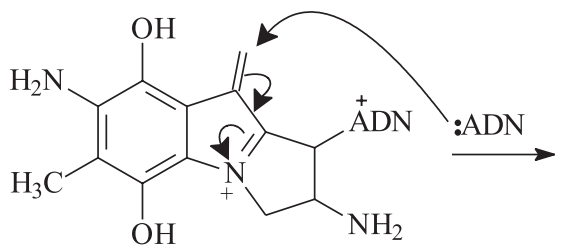<smiles></smiles>

$\mathrm{ADN}=$ ácido desoxirribonucléico

Figura 4. Ativação biorredutiva da mitomicina $C$ (1) e sua ligação ao $A D N^{30}$ 
EO9 (8), uma indoquinona análoga à mitomicina $\mathrm{C}(\mathbf{1})$, foi sintetizada em $1987^{38}$ e sua atividade antitumoral tem sido extensivamente estudada ${ }^{39,40}$.<smiles>Cn1c(/C=C/CO)c(CO)c2c1C(=O)C=C(N1CC1)C2=O</smiles>

(8)

EO9 (8) apresenta mecanismo de bioativação similar ao da mitomicina C (1). Na forma oxidada o par de elétrons não ligantes do nitrogênio do anel aziridínio encontra-se em ressonância com a carbonila da quinona. Após biorredução, a basicidade deste nitrogênio é aumentada, favorecendo, assim, a protonação da amina terciária, resultando em uma espécie altamente eletrofílica capaz de alquilar o $\mathrm{ADN}^{17,30}$ (Figura 5).

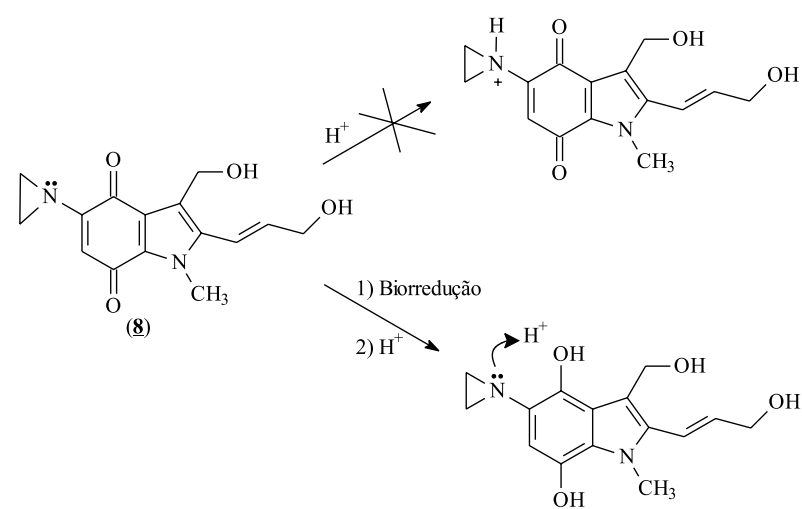

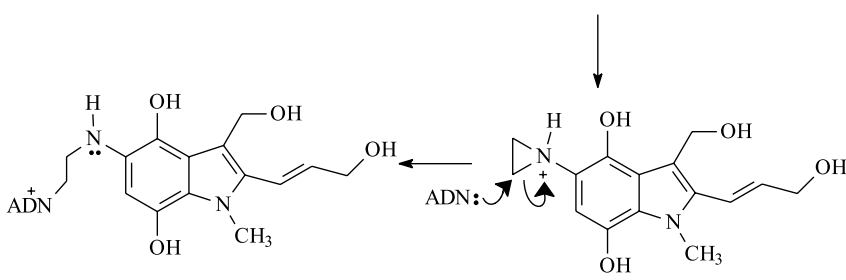

Figura 5. Ativação biorredutiva do EO9 (8) (adaptado da referência 30)

A redução de (8), além de facilitar a abertura do íon aziridínio, pode levar à ativação de uma ou das duas hidroxilas alcoólicas, por exemplo, por fosforilação in vivo, resultando em espécies alquilantes capazes de formar adutos adicionais com o $\mathrm{ADN}^{40}$. Estudos clínicos iniciais mostraram-se encorajadores ${ }^{41}$, porém, os resultados dos estudos em fase II de triagem clínica não foram favoráveis pois EO9 não mostrou atividade no tratamento de câncer de mama, estômago, pâncreas, cólon e pulmão ${ }^{42}$. Entretanto, EO9 (8) não foi testado em combinação com a radioterapia e nem com outros agentes antineoplásicos com ação sobre as células tumorais aeróbicas. Este fato pode ser responsável pela falta de atividade do EO9 (8) frente aos diversos tipos de tumores, já que este apresenta toxicidade seletiva para as células tumorais em hipóxia e pode não ser capaz de matar as células em condições de aerobiose ${ }^{43}$.

A descoberta da atividade antitumoral dos antibióticos FR900482 (9) e FR66979 (10), isolados de culturas de Streptomyces sandaensis, abriu uma nova perspectiva no tratamento do câncer ${ }^{44}$. Em estudos clínicos preliminares, FR-900482 (9) e o seu derivado acetilado FK973 (11) foram três vezes mais potentes do que a mitomicina C (1), além de terem apresentado menor toxicidade ${ }^{30,45,46}$.<smiles>[R]Oc1cc(C=O)cc2c1[C@@H](COC(N)=O)[C@]1([R])ON2CC2[M]C21</smiles>

$$
\text { FR-900482 (9) } \mathrm{R}=\mathrm{H}
$$$$
\text { FK-973 (11) } \mathrm{R}=\mathrm{Ac}
$$<smiles></smiles>

FR-66979 (10)
Apesar de a função quinona não estar presente em sua estrutura, estes antibióticos antitumorais apresentam uma similaridade estrutural com a mitomicina $C(\mathbf{1})$ e também requerem biorredução para exercerem sua atividade ${ }^{30,44-47}$. Foi demonstrado que a redução destes antibióticos não leva à formação de ânion superóxido e este fato pode explicar a baixa toxicidade apresentada por tais substâncias ${ }^{30}$. O mecanismo de biorredução de (9)-(11) envolve, primeiramente, a redução da ligação N-O da hidroxilamina hemiacetálica, conforme representado na Figura $6^{47}$.

\section{Nitroimidazóis}

No final da década de 60, Adams, Fowler e colaboradores foram os pioneiros na utilização de compostos com alta afinidade eletrônica como radiossensibilizadores (reagem com os radicais formados pela radiação) para células em hipóxia. Tais compostos teriam a capacidade de mimetizar os efeitos danosos do oxigênio ${ }^{48}$. $\mathrm{O}$ sucesso do tratamento de tumores sólidos utilizando-se o metronidazol (2)

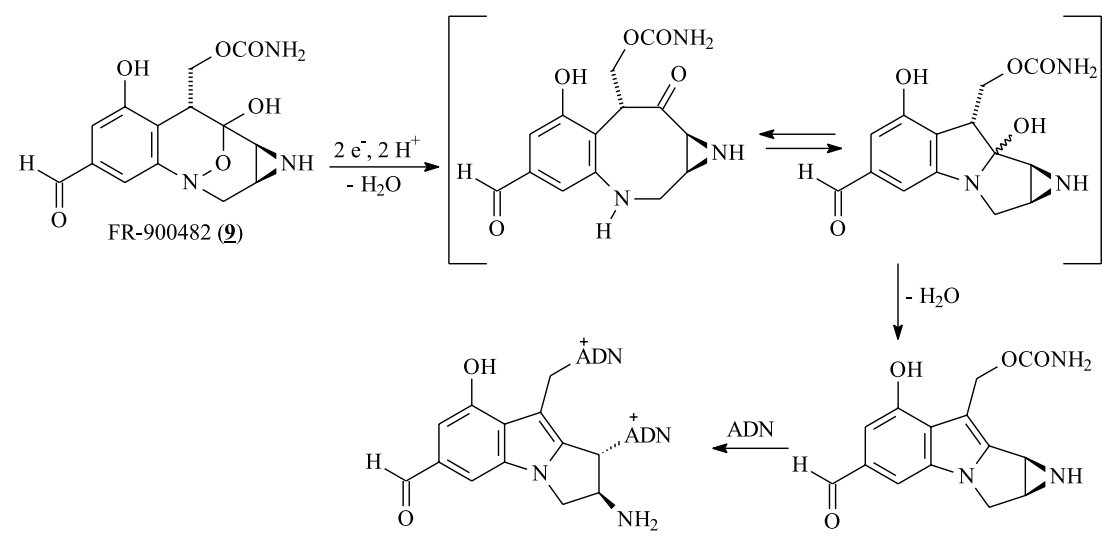


foi a confirmação in vivo daquela hipótese ${ }^{49}$. Estes compostos foram desenvolvidos como radiossensibilizadores, mas, posteriormente, foi demonstrado que a exposição prolongada das células em hipóxia aos nitroaromáticos resulta na morte seletiva de tais células, mesmo na ausência de radiaçã $0^{50}$. Isso ocorre devido à redução metabólica dos nitroaromáticos a intermediários altamente reativos, sendo que os principais responsáveis pela ação citotóxica são o ânion nitro radical $\mathrm{RNO}_{2}-$ e a hidroxilamina $\mathrm{RNHOH}^{50,51}$. Acredita-se que o radical $\mathrm{RNO}_{2}{ }^{\circ} \mathrm{H}$ atue como aceptor de elétrons (ou o ânion radical $\mathrm{RNO}_{2} \cdot$ como aceptor de elétrons e prótons), oxidando o ADN e tendo como consequiência a ruptura e destruição da dupla hélice do ácido nucléico ${ }^{51}$. Em relação à hidroxilamina, Knox e colaboradores propuseram que a capacidade deste composto em se ligar ao ADN é devida à sua acetilação in vivo e subseqüente reação do derivado $N$-acetóxido com o $\mathrm{ADN}^{52,53}$. O mecanismo de ação e as etapas de redução dos nitroimidazóis podem ser representados conforme a Figura $7^{54}$.

Pode-se notar, pela análise da Figura 7, que a presença do oxigênio pode reverter ou inibir a primeira etapa de redução. Como nas células em hipóxia a concentração de oxigênio é reduzida, a primeira etapa de redução ocorre com mais facilidade, resultando na formação de intermediários reativos. Tais derivados têm diferentes estabilidades e reatividades dependendo dos substituintes do anel aromático e do $\mathrm{pH}^{43}$.

Apesar de os nitroimidazóis, tais como o metronidazol (2) e o misonidazol (3), possuírem seletividade para células em hipóxia, eles apresentam baixa potência e, consequientemente, elevadas doses são necessárias para exercerem uma ação citotóxica ${ }^{55-58}$. Em função disto, novos compostos desta classe estão sendo estudados. O análogo (RSU 1069) (12) foi desenvolvido com o intuito de que a presença de um grupo alquilante adicional, como o aziridino, pudesse aumentar a potência deste composto ${ }^{59}$.<smiles>O=[N+]([O-])c1nccn1CC(O)CN1CC1</smiles>

O RSU 1069 (12) não somente apresentou muito maior citotoxidade do que o misonidazol (3), mas também mostrou maior seletividade para as células em hipóxia $(\sim 50-100 \text { vezes })^{57,60}$. O mecanismo de citotoxidade deste composto em condições de aerobiose é a monoalquilação do ADN pelo aziridino. Metabolismo redutivo o converte em um agente bifuncional muito mais tóxico capaz de ligação cruzada com o ADN (Figura 8). Infelizmente, no início dos estudos de triagem clínica com o RSU 1069 (12) foi demonstrada alta toxicidade gastrintestinal que impossibilitou o prosseguimento dos estudos $^{57,60}$.

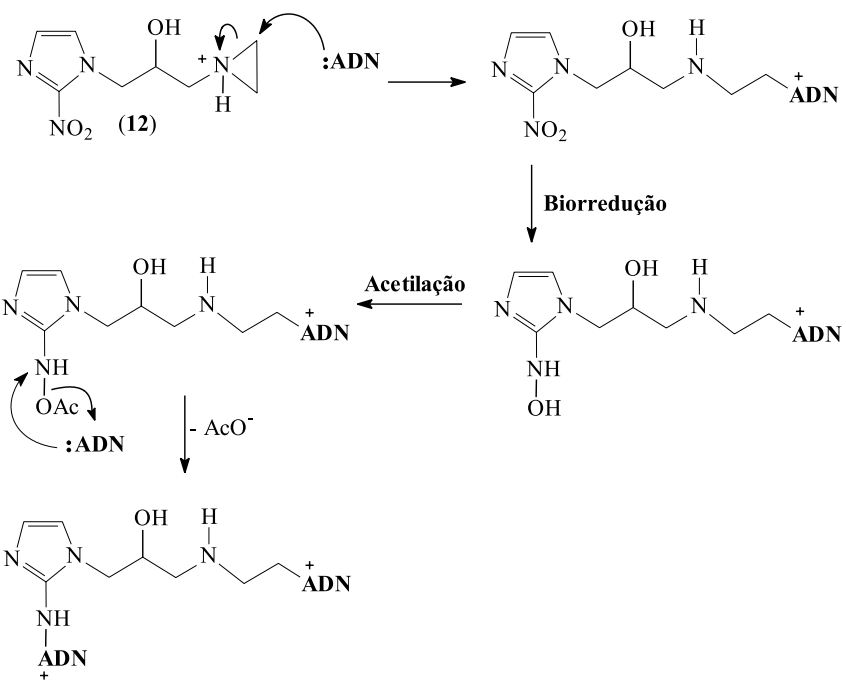

Figura 8. Ligação do RSU 1069 (12) ao ADN após biorredução(adaptado da referência 43)

Mais recentemente, o interesse foi voltado para um pró-fármaco do RSU 1069 (12), o RB 6145 (13) (Figura 9). Este composto é rapidamente convertido em (12) in vitro e in vivo e mostra atividade equivalente, mas é menos emético e apresenta pronunciada atividade por via oral ${ }^{57}$.<smiles>CCC1(Br)CCN(CC(O)Cn2ccnc2[N+](=O)[O-])CC1</smiles>
(13)

Figura 9. Conversão do RB 6145 (13) em RSU 1069 (12) in vivo ${ }^{43}$

O enantiômero R do RB 6145 (13) encontrava-se em estudos clínicos ${ }^{61}$, porém os testes foram interrompidos devido à toxicidade para a retina de roedores e macacos. Investigações adicionais mostraram que tal toxicidade está relacionada à hipóxia fisiológica na retina ${ }^{62}$.

\section{Di- $N$-óxidos-benzotriazinas}

A toxicidade seletiva da tirapazamina (4) (1,4-di- $N$-óxido-3amino-1,2,4-benzotriazina, SR 4233) frente às células tumorais em hipóxia foi descoberta por Brown e colaboradores em $1986^{25}$.

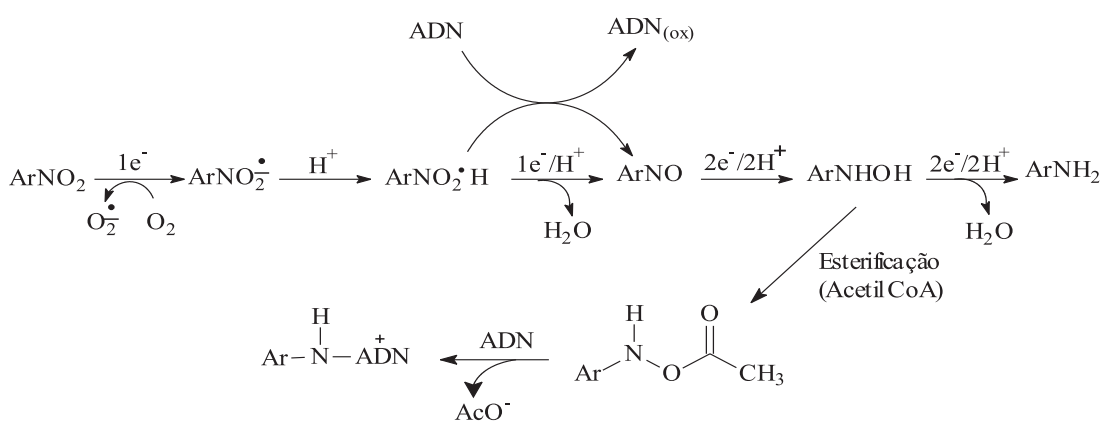

Figura 7. Mecanismo de ação de substâncias nitroaromáticas após biorredução (adaptado das referências 51 e 52) 
A tirapazamina (4) é um excelente substrato para várias redutases intracelulares, as quais a reduzem pela adição de um elétron, resultando na formação de um intermediário radicalar altamente reativo. Este radical formado pode, então, remover um átomo de hidrogênio de biomoléculas. Se esta biomolécula for o ADN, o resultado é a quebra da dupla fita, o que leva a aberrações nos cromossomas e morte celular. Reduções adicionais a mono $N$-óxido (redução por dois elétrons) ou redução por quatro elétrons origina produtos não tóxicos (Figura 10) $)^{63,64}$.<smiles>Nc1n[n+]([O-])c2ccccc2[n+]1[O-]</smiles>

tirapazamina (4)
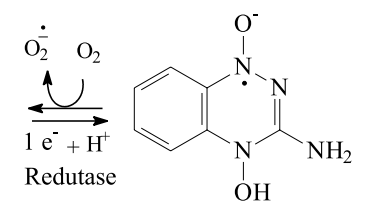

espécie radicalar

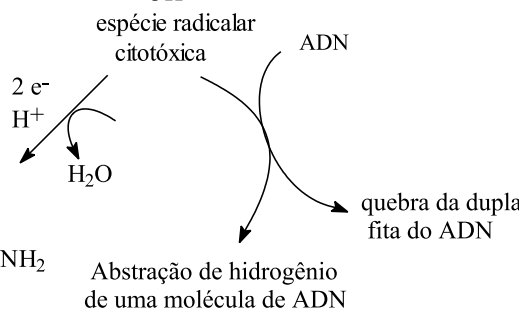

inativo

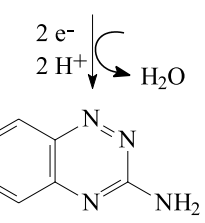

inativo
Figura 10. Mecanismo proposto para ativação biorredutiva da tirapazamina $(4)^{63}$

Como a tirapazamina (4) é seletivamente tóxica para as células em hipóxia não é de se esperar que ela seja capaz de matar todas as células tumorais, pois muitas dessas células são bem oxigenadas. Estudos de triagem clínica já demonstraram que a tirapazamina (4) não é capaz de erradicar todo o tumor ${ }^{65}$. Entretanto, quando combinada com a radioterapia, a tirapazamina (4) é altamente efetiva e potencializa a morte das células tumorais. Ela é, também, extremamente efetiva para a potencialização da atividade antitumoral da cisplatina. Foi demonstrado que a incubação de células sob condições de hipóxia na presença da tirapazamina (4), antes do tratamento com cisplatina, aumenta as ligações cruzadas entre a cisplatina e o $\mathrm{ADN}$, sugerindo que (4) inibe ou retarda o reparo do $\mathrm{ADN}^{65}$.

Atualmente, a tirapazamina (4) encontra-se em fase final de triagem clínica e os resultados mostram que esta, combinada com a cisplatina, foi capaz de aumentar, significativamente, o tempo médio de sobrevida dos pacientes ${ }^{13,64}$.

\section{OUTRAS CLASSES DE AGENTES BIORREDUTÍVEIS}

Várias outras classes de agentes biorredutíveis vêm sendo descritas $^{66-74}$ e uma delas é a dos $N$-óxidos de alquilaminoantraquinonas, representada pela AQ4N (1,4-bis-([2-(dimetilamino- $N$ óxido)etil]amino)-5,8-diidroxiantraceno-9,10-diona) (14). Após ativação redutiva, AQ4N (14) é convertida na AQ4 (15) (Figura 11), a qual tem alta afinidade pelo ADN e inibe a enzima topoisomerase II. A interação de AQ4 (15) com o ADN é facilitada pela sua estrutura planar, deficiente de elétrons, capaz de intercalar entre pares de base adjacentes do ADN. Este complexo é estabilizado por interações

eletrostáticas e ligações de hidrogênio com os grupos fosfato da desoxirribose ${ }^{64,75,76}$.<smiles>CN(C)CCNc1ccc(NCCN(C)C)c2c1C(=O)c1c(O)ccc(O)c1C2=O</smiles>

Figura 11. Estruturas químicas da $A Q 4 N$ (14) e do seu produto de redução $A Q 4(15)^{75}$

A combinação de AQ4N (14) com a radioterapia e/ou com outros fármacos antitumorais tem fornecido resultados promissores ${ }^{77}$. Atualmente, a AQ4N (14) está em estudos de triagem clínica ${ }^{62}$.

Uma outra classe de agentes biorredutíveis que não poderia deixar de ser citada é a das mostardas nitroaromáticas. Em 1986, Denny e Wilson lançaram as bases teóricas do planejamento racional de mostardas nitroaromáticas como potenciais agentes citotóxicos seletivos para células em hipóxia ${ }^{18}$. A partir de então, várias mostardas nitroaromáticas, como as mostardas (16) e (17), vêm sendo extensivamente estudadas como pró-fármacos ${ }^{18,20,78-84}$.<smiles>Cn1cnc([N+](=O)[O-])c1C[N+](C)(Cl)CCCl</smiles>

SN $25341(\mathbf{1 6})$<smiles>NC(=O)c1cc(N(CCCl)CCCl)c([N+](=O)[O-])cc1[N+](=O)[O-]</smiles>

SN $23862(\mathbf{1 7})$
A ativação da mostarda 2-nitroimidazólica (16) ocorre a partir de uma fragmentação do ânion nitro radical formado por redução monoeletrônica, originando um radical do tipo benzílico, via transferência de elétron intramolecular ${ }^{84}$. Esta proposta mecanística é análoga à bem conhecida fragmentação redutiva dos haletos 2nitrobenzílicos ${ }^{85-87}$ (Figura 12).<smiles>CCCc1ccccc1CCl</smiles>

Figura 12. Fragmentação redutiva de haletos de nitrobenzila ${ }^{82}$

Após sofrer redução, a mostarda (16) libera, então, a mecloretamina (18), mostarda altamente tóxica para as células tumorais (Figura 13).

No caso da mostarda (17), a introdução dos grupos nitro (retiradores de elétrons) no anel aromático desativa a mostarda por deslocalização do par de elétrons do nitrogênio amínico. Em células em hipóxia, o grupo nitro é reduzido a hidroxilamino e/ou amino (doador de elétrons), aumentando a densidade eletrônica no anel aromático, com conseqüente ativação da mostarda nitrogenada ${ }^{18}$. 


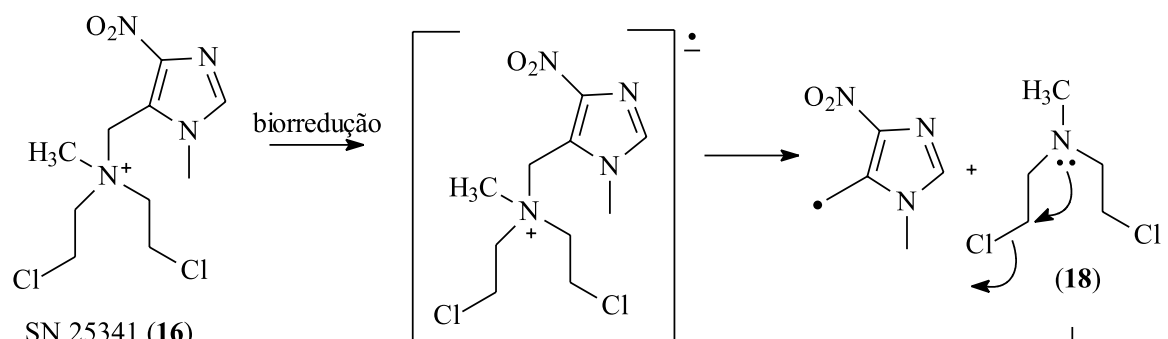

SN $25341(\underline{\mathbf{1 6}})$

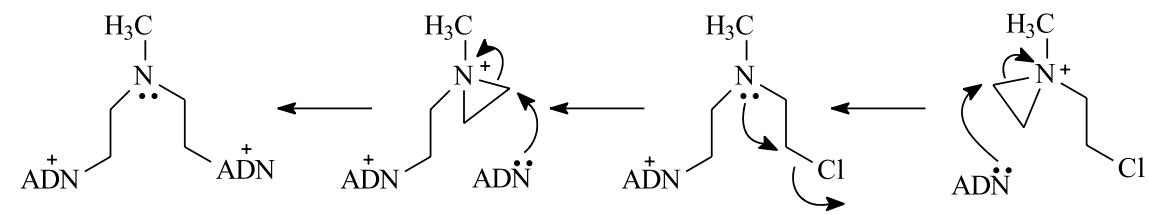

Figura 13. Fragmentação redutiva e mecanismo de ação da mostarda (16)(adaptado das referências 82,84)

O estudo do metabolismo redutivo da mostarda (17) mostrou que a redução ocorria exclusivamente no grupo nitro localizado em orto em relação à mostarda, resultando no derivado 4-hidroxilamino (19) e/ou amino (20). Porém, estes metabólitos sofrem rápida ciclização intramolecular, originando a tetraidroquinoxalina (21) que possui atividade muito inferior à de (19) e (20) (Figura 14) ${ }^{81}$.
SN $23862(17)$<smiles>C[C@H]1C[C@@H](C)C1c1cc(C(N)=O)c([N+](=O)[O-])cc1[N+](=O)[O-]</smiles><smiles>[R]N1CCN(CCCl)c2cc(C(N)=O)c([N+](=O)[O-])cc2N1CCCl</smiles>

$\mathrm{R}=\mathrm{OH}(\mathbf{1 9})$ $\mathrm{R}=\mathrm{H}(\mathbf{2 0})$
Figura 14. Biorredução da mostarda (17) (adaptado da referência 43)

A formação de (21) constituiu, então, um empecilho na utilização da mostarda (17) em estudos adicionais. Entretanto, este problema pode ser solucionado quando Anlezark e colaboradores isolaram e purificaram uma nitrorredutase de Escherichia coli capaz de reduzir seletivamente o grupo nitro de (17), localizado na posição dois, a hidroxilamino ${ }^{88,89}$. A redução deste grupo nitro leva à ativação da mostarda e evita a formação de (21). A partir de então, a mostarda (17) vem sendo estudada utilizando-se o sistema ADEPT ("AntibodyDirected Enzyme Prodrug Therapy” ${ }^{89}$. Esta terapia fundamenta-se na ativação enzimática de um pró-fármaco usando um conjugado anticorpo-enzima (AEC) específico para antígenos presentes na membrana das células tumorais ${ }^{90,91}$. Neste caso, a enzima utilizada é a nitrorredutase de E. coli. Em linhas gerais, após administração e distribuição pelo organismo, o AEC liga-se às células tumorais e, em seguida, administra-se a mostarda (17) (pró-fármaco) ${ }^{90}$. Esta é, então, reduzida pela nitrorredutase acoplada ao anticorpo, liberando a mostarda na sua forma ativa (Figura 15), na região do tumor ${ }^{89}$.

Além das mostardas nitroaromáticas, outras mostardas biorredutíveis vêm sendo desenvolvidas ${ }^{30}$, como, por exemplo, complexos de cobalto com mostardas alifáticas ${ }^{30,92,93}$. Coordenação do nitrogênio da mostarda com Co(III) torna o composto inativo. A alta energia de estabilização dos complexos de Co(III) (configuração d ${ }^{6}$,

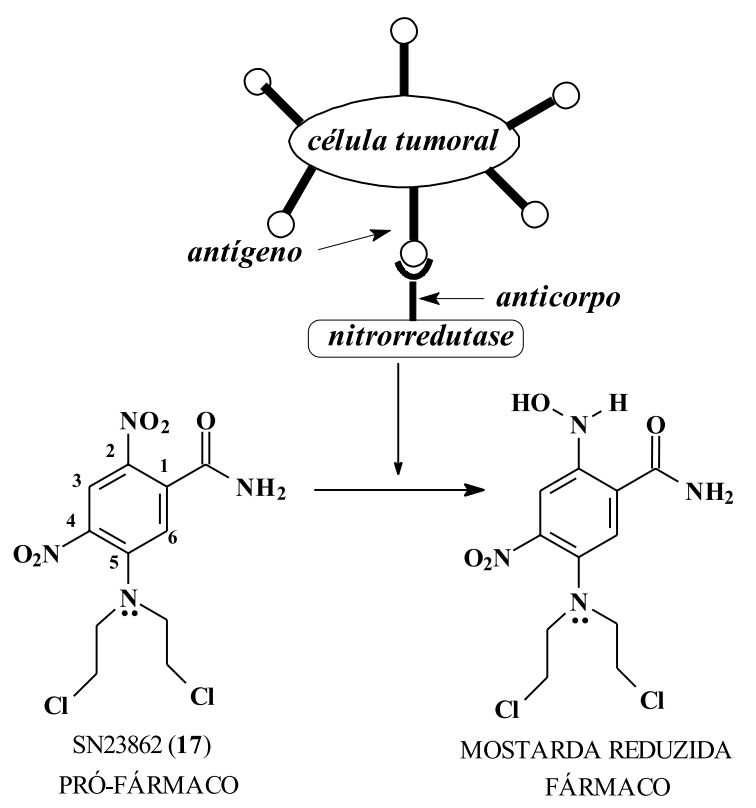

Figura 15. Representação esquemática da terapia ADEPT, utilizando-se nitrorredutase de E. coli e a mostarda SN23862 (17)(adaptado da ref. 91)

baixo spin) faz com que estes complexos sejam cineticamente inertes e, portanto, as reações de hidrólise com conseqüiente deslocamento da mostarda nitrogenada são extremamente lentas. A redução monoeletrônica dos complexos de $\mathrm{Co}(\mathrm{III})$ nas regiões de hipóxia dos tumores sólidos é possível devido ao fato de que o potencial de redução $\mathrm{Co}(\mathrm{III})-\mathrm{Co}(\mathrm{II})$ está dentro da faixa ideal para a atividade das enzimas redutases. O complexo de $\mathrm{Co}$ (II) resultante sofre facilmente reações de substituição pela água, liberando a mostarda nitrogenada ativa e $\left[\mathrm{Co}\left(\mathrm{H}_{2} \mathrm{O}\right)_{6}\right]^{2+}(\text { Figura 16) })^{30,92,93}$.

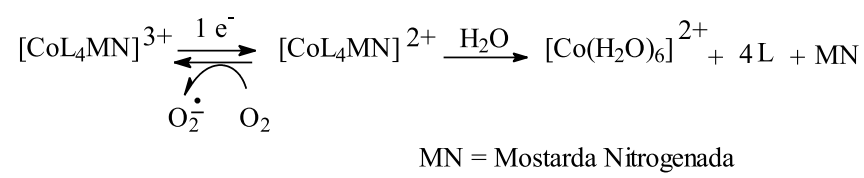

Figura 16. Biorredução de complexos de cobalto (III $)^{92}$ 
Vários complexos deste tipo foram sintetizados e testados e, até o momento, o que apresentou melhor atividade e seletividade in vitro foi o complexo $(\mathbf{2 2})^{30,92,93}$.

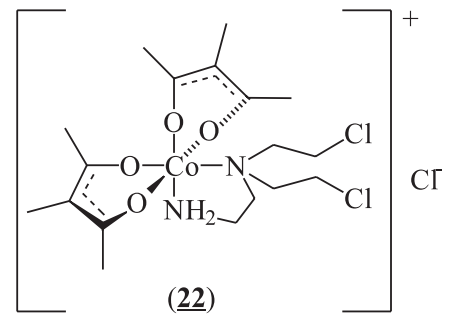

\section{CONSIDERAÇÕES FINAIS}

O conceito de bioativação como um mecanismo para a ação de fármacos vem sendo cada vez mais difundido na busca de fármacos menos tóxicos e mais seletivos. Uma área particularmente fascinante é o planejamento e o desenvolvimento de pró-fármacos do tipo bioprecursores biorredutíveis, os quais só se tornam ativos após redução in vivo. A descoberta da existência de células em condições de hipóxia nos tumores sólidos pode ser explorada no desenvolvimento desses pró-fármacos biorredutíveis. Apesar dos vários exemplos de substâncias que apresentam atividade antitumoral após sofrerem biorredução, esta é, ainda, uma área pouco estudada, porém muito promissora. Acredita-se que o ajuste adequado das propriedades eletrônicas e fisico-químicas de tais substâncias possa levar ao desenvolvimento de novos fármacos antitumorais úteis na quimioterapia dos tumores sólidos.

\section{AGRADECIMENTOS}

Os autores expressam seus agradecimentos à Profa. M. A. F. Prado, da Faculdade de Farmácia (UFMG), pela leitura crítica do manuscrito e sugestões.

\section{REFERÊNCIAS}

1. Katzung, B. C.; Farmacologia Básica e Clínica, Guanabara Koogan: Rio de Janeiro, 1994, p. 579.

2. Molinoff, P. B.; Ruddon, R. W., eds.; Goodman \& Gilman As Bases Farmacológicas da Terapêutica, Mc Graw Hill: Rio de Janeiro, 1996, p. 909-949.

3. Kennedy, K. A.; Teicher, B. A.; Rockwell, S.; Sartorelli, A.C.; Biochem. Pharmacol. 1980, 29, 1.

4. Foye, W. O.; Lemke, T. L.; Williams, D. A.; Principles of Medicinal Chemistry, Williams \& Wilkings: Baltimore, 1995, p. 822-845.

5. Jain, R. K.; Scientific Amer. 1994, 271, 42.

6. Jain, R. K.; J. Nat. Cancer Inst. 1989, 81, 570.

7. Vaupel, P.; Kallinowski, F.; Okunieff, P.; Cancer Res. 1989, 49, 6449.

8. Molena, G.; Meijer, D. K. F.; Leij, L. F. M. H.; Biochem. Pharmacol. 1998, $53,1939$.

9. Brown, J. M.; Giaccia, A. J.; Cancer Res. 1998, 58, 1408.

10. Hellman, S.; Rosenberg, S. A.; Cancer Principles and Practise of Oncology, J. B. Lippincott: Philadelphia, 1989.

11. Wolff, M. E.; Burger's Medicinal Chemistry, John Wiley \& Sons: New York, 1981, p.11-45.

12. Denny, W. A.; The Lancet Oncol. 2000, 1, 25.

13. Brown, J. M.; Cancer Res. 1999, 59, 5863.

14. Teicher, B. A.; Lazo, J. S.; Sartorelli, A. C.; Cancer Res. 1981, 41, 73

15. Lin, A. J.; Cosby, L. A.; Shansky, C. W.; Sartorelli, A. C.; J. Med. Chem. 1972, 15, 1247

16. Wermuth, C. G.; The Practice of Medicinal Chemistry, Academic Press: London, 1996, p. 697-716.

17. Silverman, R. B.; The Organic Chemistry of Drug Design and Drug Action, Academic Press: London, 1992.
18. Denny, W. A.; Wilson, W. R.; J. Med. Chem. 1986, 29, 879.

19. Lin, A. J.; Sartorelli, A. C.; Biochem. Pharmacol. 1976, 25, 206.

20. Palmer, B. D.; Wilson, W. R.; Cliffe, S.; Denny, W. A.; J. Med. Chem. 1992, $35,3214$.

21. Rockweel, S.; Kennedy, K. A.; Sartorelli, A. C.; Int. J. Radiat. Oncol. Biol. Phys. 1982, 8, 753.

22. Kennedy, K. A.; Rockwell, S.; Sartorelli, A. C.; Cancer Res. 1980, 40, 2356

23. Varghese, A. J.; Gulyas, S.; Mohindra, J. K.; Cancer Res. 1976, 36, 3761.

24. Moore, H. W.; Science 1977, 197, 527.

25. Zeman, E. N.; Brown, J. M.; Lemmon, M. J.; Hirst, V. K.; Lee, W. W.; Int. J. Radiat. Oncol. Biol. Phys. 1986, 12, 1239.

26. Beigleiter, A.; Front. Biosci. 2000, 5, 153.

27. Wakaki, S.; Marumo, H.; Tomloka, K.; Shimizu, G.; Kato, E.; Kamada, H.; Kudo, S.; Fujimoto, Y.; Antibiot. Chemother. 1958, 8, 228.

28. Sartorelli, A. C.; Cancer Res. 1988, 48, 775.

29. Bizanek, R.; Chowdary, D.; Arai, H.; Kasai, M.; Hughes, C. S.; Sartorelli, A. C.; Rockwell, S.; Tomasz, M.; Cancer Res. 1993, 53,5127.

30. Rajski, S. R.; Williams, R. M.; Chem. Rev. 1998, 98, 2723.

31. Kinoshita, S.; Uzu, K.; Nakano, K.; Shimizu, M.; Takahashi, T.; Matsui, M.; J. Med. Chem. 1971, 14, 103.

32. Stringfellow, D. A.; Schurig, J. E.; Cancer Treat. Rev. 1987, 14, 291.

33. Iyengar, B. S.; Remers, W. A.; Catino, J. J.; J. Med. Chem. 1989, 32, 1866.

34. Arai, H.; Kanda, Y.; Ashizawa, T.; Morimoto, M.; Gomi, K.; Kono, M.; Kasai, M.; J. Med. Chem. 1994, 37, 1794.

35. Denny, W. A.; Wilson, W. R.; Expert Opin. Invest. Drugs 2000, 12, 2889.

36. Foley, H. T.; Shnider, B. I.; Gold, G. L.; Matias, P. I.; Colsky, J.; Miller, S. P.; Cancer Chemother. Rep. 1967, 51, 283.

37. Haffy, B. G.; Son, Y. H.; Papac, R.; Fischer, D.; Wilson, L. D.; Rockwell, S.; Sartorelli, A. C.; Sasaki, C.; Fischer, J. J.; Int. J. Radiat. Oncol. Biol. Phys. Supplement 1 1996, 36, 331.

38. Oosterveen, E. A.; Speckmap, W. N.; Tetrahedron 1987, 43; 255.

39. Smitskamp-Wilms, E., Hendriks, H. R.; Peters, G. J.; Gen. Pharmacol. 1996, 27, 421

40. Bailey, S. M.; Wyatt, M. D.; Friedlos, F.; Hartley, J. A.; Knox, R. J.; Lewis, A. D.; Workman, P.; Br. J. Cancer 1997, 76, 1596.

41. Schellens, J. H. M.; Planting, A. S. T.; Van Acker, B. A. C.; Loos, W. J.; De Boer-Dennert, M.; Van der Burg, M. E. L.; Koier, I.; Krediet, R. T.; Stoter, G.; Verweij, J.; J. Nat. Cancer Inst. 1994, 86, 906.

42. Wanders, J.; Pavlidis, N.; Gamucci, T.; Huinink, W. W. T.; Kirix, L.; Wolff, I.; Verweij, J.; Eur. J. Cancer 1995, 31, 565.

43. Rauth, A. M.; Melo, T.; Misra, V.; Int. J. Radiat. Oncol. Biol. Phys. 1998, 42,755 .

44. Fukuyama, T.; Goto, S.; Tetrahedron Lett. 1989, 30, 6491.

45. Paz, M. M.; Sigurdsson, S. T.; Hopkins, P. B.; Bioorg. Med. Chem. 2000, 8, 173.

46. Rajski, S. R.; Williams, R. M.; Bioorg. Med. Chem. 2000, 8, 1331.

47. Huang, H.; Rajski, S. R.; Williams, R. M.; Hopkins, P.; Tetrahedron Lett. 1994, 35, 9669.

48. Adams, G. E.; Br. Med. Bull. 1973, 29, 48.

49. Rauth, A. M.; Kaufman, K.; Br. J. Radiat. 1975, 48, 209.

50. Taylor, Y. C.; Rauth, A. M.; Cancer Res. 1978, 38, 2745.

51. La-Scalea, M. A.; Rev. Farm. Bioquím.Univ. São Paulo 1998, 34, 59.

52. Knox, R. J.; Friedlos, F.; Jarman, M.; Roberts, J. J.; Biochem. Pharmacol. 1988, 37, 4661.

53. Knox, R. J.; Friedlos, F.; Marchbank, T.; Roberts, J. J.; Biochem. Pharmacol. 1991, 42, 1691.

54. Adams, G. E.; Radiat. Res. 1992, 132, 129.

55. Agrawal, K., C.; Bears, K. B.; Sehgal, R. K.; J. Med. Chem. 1979, 22, 583.

56. Sakaguchi, M.; Webb, M. W.; Agrawal, K. C.; J. Med. Chem. 1982, 25, 1339.

57. Hay, M. P.; Wilson, W. R.; Moselen, J. W.; Palmer, B. D.; Denny, W. A.; J. Med. Chem. 1994, 37, 381.

58. Hay, M. P.; Lee, H. H.; Wilson, W. R.; Roberts, P. B.; Denny, W. A.; J. Med. Chem. 1995, 38, 1928 .

59. Jenkins, T. C.; Naylor, M. A.; O’Neill, P.; Threadgill, M. D.; Cole, S.; Stratford, I. J.; Adams, G. E.; Fielden, E. M.; Suto, M. J.; Stier, M. A.; J. Med. Chem. 1990, 33, 2603.

60. Naylor, M. A.; Threadgill. M. D.; Webb, P.; Stratford, I. J.; Stephens, M. A.; Fielden, E. M.; J. Med. Chem. 1992, 35, 3573.

61. Naylor, M. A.; Threadgill, M. D.; Showalter, H. D. H.; Stratford, I. J.; Stephens, M. A.; Fielden, E. M.; Adams, G. E.; Drug Des. Discovery 1993, $10,249$.

62. Lee, A. E.; Wilson, W. R.; Toxicol. Appl. Pharmacol. 2000, 163, 50.

63. Brown, J. M.; Br. J. Cancer 1993, 67, 1163.

64. Brown, J. M.; Mol. Med. Today 2000, 6, 157.

65. Kovacs, M. S.; Hocking, D. J.; Evans, J. W.; Siim, B. G.; Wouters, B. G.; Brown, J. M.; Br. J. Cancer 1999, 80, 1245 
66. Wilson, W. R.; Anderson, R. F.; Denny, W. A.; J. Med. Chem. 1989, 32, 23.

67. Wilson, W. R.; Thompson, L. H.; Anderson, R. F.; Denny, W. A.; J. Med. Chem. 1989, 32, 31.

68. Denny, W. A.; Atwell, G. J.; Anderson, R. F.; Wilson, W. R.; J. Med. Chem. 1990, 33, 1288.

69. Mulcahy, R. T.; Gipp, J. J.; Schmidt, J. P.; Joswig, C.; Borch, R. F.; J. Med. Chem. 1994, 37, 1610 .

70. Tercel, M.; Wilson, W. R.; Denny. W. A.; J. Med. Chem. 1995, 38, 1247.

71. Hay, M. P.; Lee, H. H.; Wilson, W. R.; Roberts, P. B.; Denny, W. A.; J. Med. Chem. 1995, 38, 1928.

72. Lee, H. H.; Wilson, W. R.; Ferry, D. M.; Zijl, P. V.; Pullen, S. M.; Denny, W. A.; J. Med. Chem. 1996, 39, 2508.

73. Siim, B. G.; Atwell, G. J.; Anderson, R. F.; Wardman, P.; Pullen, S. M.; Wilson, W. R.; Denny, W. A.; J. Med. Chem. 1997, 40, 1381.

74. Siim, B. G.; Hicks, K. O.; Pullen, S. M.; Zijl, P. L.; Denny, W. A.; Wilson, W. R.; Biochem. Pharmacol. 2000, 60, 969.

75. McKeown, S. R.; Hejmadi, M. V.; Mcintyre, I. A.; Mcaleer, J. J. A.; Patterson, L. H.; Br. J. Cancer 1995, 72, 76.

76. Patterson, L. H.; Mckeown, S. R.; Br. J. Cancer 2000, 83, 1589.

77. Hejmadi, M. V.; McKeown, S. R.; Friery, O. P.; Mcintyre, I. A.; Patterson L. H.; Hirst, D. G.; Br. J. Cancer 1996, 73, 499.

78. Palmer, B. D.; Wilson, W. R.; Pullen, S. M.; Denny, W. A.; J. Med. Chem. 1990, 33, 112 .

79. Atwell, G. J.; Sykes, B. M.; O’Connor, C. J.; Denny, W. A.; J. Med. Chem. 1994, 37, 371 .

80. Palmer, B. D.; Wilson, W. R.; Atwell, G. J.; Schultz, D.; Xu, X. Z.; Denny, W. A.; J. Med. Chem. 1994, 37, 2175.
81. Palmer, B. D.; Zijl, P.; Denny, W. A.; Wilson, W. R.; J. Med. Chem. 1995, $38,1229$.

82. Tercel, M.; Wilson, W. R.; Anderson, R. F.; Denny, W. A.; J. Med. Chem 1996, 39, 1084.

83. Lee, H. H.; Palmer, B. D.; Wilson, W. R.; Denny, W. A.; Bioorg. Med. Chem. Lett. 1998, 8, 1741.

84. Wilson, W. R.; Ferry, D. M.; Tercel, M.; Anderson, R. F.; Denny, W. A.; Radiat. Res. 1998, 149, 237.

85. Neta, P.; Behar, D.; J. Am. Chem. Soc. 1980, 102, 4798.

86. Bays, J. P.; Blumer, S. T.; Tosh, S. B.; Behar, D.; Neta, P.; J. Am. Chem. Soc. 1983, 105, 320

87. Morris, R. K.; Barker, S. D.; Neta, P.; J. Am. Chem. Soc. 1984, 106, 3140.

88. Anlezark, G. M.; Melton, R. G.; Sherwood, R. F.; Coles, B.; Friedlos, F.; Knox, R. J.; Biochem. Pharmacol. 1992, 44, 2289.

89. Anlezark, G. M.; Melton, R. G.; Sherwood, R. F.; Wilson, W. R.; Denny, W. A.; Palmer, B. D.; Knox, R. J.; Friedlos, F.; Williams, A.; Biochem. Pharmacol. 1995, 50, 609.

90. Bagshawe, K. D.; Sharma, S. K.; Springer, C. J.; Rogers, G. T.; Ann. Oncol. 1994, 5, 879 .

91. Chin, C. M.; Ferreira, E. I.; Quim. Nova 1999, 22, 75.

92. Ware, D. C.; Palmer, B. D.; Wilson, W. R.; Denny, W. A.; J. Med. Chem. 1993, 36, 1839.

93. Ware, D. C.; Palmer, H. R.; Pruijn, F. B.; Anderson, R. F.; Brothers, P. J.; Denny, W. A.; Wilson, W. R.; Anti-Cancer Drug Des. 1998, 13, 81 . 